\title{
Introduction of a novel therapeutic option for atrophic acne scars: saline injection therapy
}

\author{
Nooshin Bagherani ${ }^{1 *}$ and Bruce R Smoller ${ }^{2 *}$ \\ ${ }^{1}$ Dermatologist at Dr. Nooshin Bagheran's Office, Iran \\ ${ }^{2}$ Professor and Chair, Department of Pathology, Professor, Department of Dermatology, University of Rochester, School of Medicine and Dentistry, USA
}

\begin{abstract}
Introduction and objectives: Atrophic acne scars are extremely bothersome to patients and often challenging to treat. For their treatment, topical, physical, surgical, and light modalities, alone or in combination have been administered with variable results.Herein, for the first time we introduced saline injection therapy as a novel effective therapeutic option for atrophic acne scars.

Material and methods: We administered $0.9 \%$ sodium chloride solution in this study. After local anesthesia with lidocaine, saline was injected intra- and subdermally in the regions of acne scars and skin pores, so that it over-corrected the scars and widespread to the neighboring regions. The results were objectively and subjectively assessed by a visual score.

Results and conclusions: Our study revealed that the response to saline therapy was significant in large pores and all types of atrophic acne scars. In comparison with severe acne scars, the patients with mild and moderate scars showed better responses. This study showed that saline injection therapy can be an effective therapeutic option for atrophic acne scars with no significant side effects.
\end{abstract}

\section{Introduction}

Acne is one of the most common skin disorders seen in adolescents and adults. Its prevalence in adolescents varies from 35\% to more than $90 \%$ [1]. Post-acne erythema, dyschromia, and scarring are complications of inflammatory and nodulocystic acne eruptions, among which scarring results in the most potentially permanent devastating effects [2].

Regarding texture, acne scars are divided into scars with normal texture and scars with atrophic texture [3]. The scars with normal texture include elevated, hypertrophic scars and keloids [3], and ones with atrophic texture consist of icepick, boxcar and rolled scars $[3,4]$. Topical, physical, surgical, and light modalities, alone or in combination have been administered in the treatment of acne scars with variable results [3-5].

Herein, for the first time weintroduced saline injection therapy as a novel effective therapeutic option for the atrophic acne scars.

\section{Materials and methods}

In this study, patients with atrophic acne scars were selected from among patients referred to Dr. Nooshin Bagherani's private dermatology office. Patients with hypertension, cardiovascular diseases, and history of hypersensitivity reactions to lidocaine have been excluded from the study. After describing the process of the study, a written consent was acquired from them.

We administered $0.9 \%$ sodium chloride solutionin this study. After local anesthesia with lidocaine, the saline was injected intra- and sub-dermally in the regions of acne scars and skin pores with a $5 \mathrm{cc}$ syringe and 27-gauge needle. The amount of saline for injection was determined based on the severity of scars, so that it over-corrected the scars and spread to the neighboring regions. In every session, before the procedure, photographs of the patients were taken with their permission. This process was done weekly for 5 weeks and topical alpha hydroxy acid-containing agents were prescribed beside this procedure.

In every session, we objectively and subjectively assessed the results of treatment regarding the type of acne scar. In this study, for evaluating the response we used a visual score including scores1-3 as a poor response, 4-6 as a moderate response, 7-9 as a good response, and $10-12$ as a significant response (Table 1). The subjective evaluation was acquired by questioning about the general success of treatment from the patients. For the objective evaluation, the photographs were observed. This observation included the assessment of response according to the type of acne scar.

Table 1. Visual scoring for subjectively and objectively assessing response to the treatment.

\begin{tabular}{|l|l|}
\hline Score & Description \\
\hline $1-3$ (poor response) & No or very mild satisfaction with the treatment \\
4-6 (moderate response) & Mild to moderate satisfaction with the treatment \\
7-9 (good response) & Fair to good satisfaction with treatment \\
$10-12$ (significant response) & Excellent to complete satisfaction with the treatment \\
\hline
\end{tabular}

Correspondence to: PNooshin Bagherani, MD, Dermatologist at Dr. Nooshin Bagheran's Office, Taha Physicians' building, P.O.Box: 6414715878, Khoramshahr, Khuzestan Province, Iran; E-mail: nooshinbagherani@yahoo.com

Bruce R Smoller, MD, Professor and Chair, Department of Pathology, Professor, Department of Dermatology, University of Rochester, School of Medicine and Dentistry, USA, E-mail: bsmoller@me.com

Received: November 23, 2015; Accepted: December 15, 2015; Published: December 19, 2015 
For every case, the objective and subjective scores in every session and the mean of these scoresafter the last session were considered for assessment of the response. At the end, the data was analyzed by the SPSS software.

\section{Results}

Among the 12 cases studied, $10(83.3 \%)$ cases were female and 2 (16.7\%) cases were male. The minimum and maximum ages of patients were 18 and 56 years, respectively. Among the subjects, 6 (50\%) and $1(8.3 \%)$ patients had unsuccessfully undergone laser therapy and microdermabrasion for treating their acne scars, respectively. Isotretinoin was simultaneously administered in 4 (33.3) patients with the active acne.

Ice pick, boxcar and rolled scars, and large pores were seen in 4 (33.3\%), $10(83.3 \%), 8(66.7 \%)$ and $6(50 \%)$ of patients, respectively. The patients had Fitzpatrick's skin types of III and IV.

After the first and fifth sessions, the mean subjective response scores were $7.1 \pm 1.2$ and $10.2 \pm 1.08$, respectively. In the objective assessments, the mean score responses were $6.7 \pm 1.5,7.2 \pm 2.02,7.0$ \pm 1.8 , and $7.2 \pm 1.01$ for the ice pick, boxcar, rolled scars and pores, respectively. After the fifth session, these scores were $10.0 \pm 0.9,10.9 \pm$ $1.01,10.4 \pm 1.3$, and 10.3 \pm 1.3 , respectively (Figure 1).The response was better in the acne patients simultaneously receiving oral isotretinoin therapy in comparison with other cases, but it was not statistically significant.

No statistically significant difference in the response was reported as a function of age, gender, skin type, and severity of acne scars.

The patients reported skin lightening and tightening, decreasing of wrinkles and eminent cheeks as side profits of this procedure. Interestingly, one patient with hirsutism reported a decrease in the hair growth rate in the site of treatment. Mild ecchymosis was the only side effect and was seen in about one third of patients.
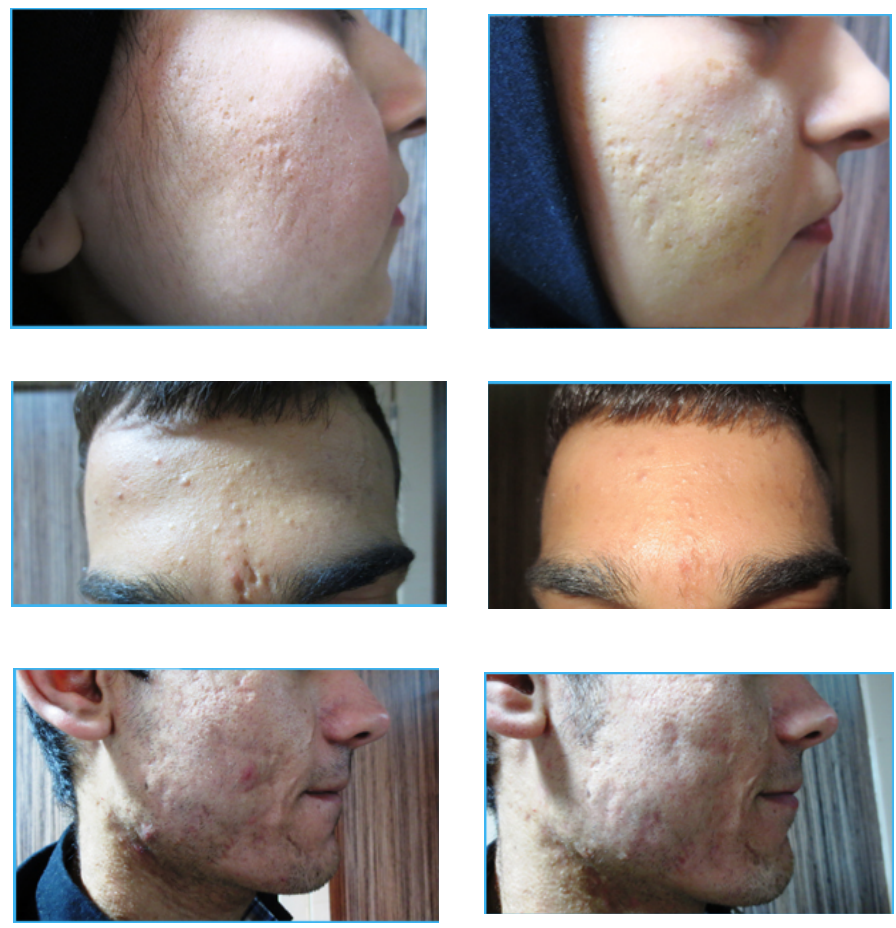

Figure 1. The efficacy of saline therapy in treating: A- icepick scars, B-boxcar scars, and C- rolled scars.

\section{Discussion}

In the treatment of atrophic acne scars, superficial irregularities can be managed by resurfacing techniques $[1,3,4]$. The ablative lasers are the gold standard in the treatment of acne scars with an efficacy of $25 \%$ to $90 \%$ [6]. Better results can be achieved with using high energy levels, but in parallel, their side effects increase [2]. In comparison with the ablative lasers, the non-ablative ones have lower side effects $[4,7,8]$. They can induceneo-collagen formation, remodel the dermis $[6,8]$, and weaken tethering adhesions [3] through transmitting energy to the dermis without damaging the skin surface [7]. Clinical improvement of $40 \%$ to $50 \%$ has been reported with these lasers [6]. Side effects of lasers include acneiform eruptions [7], postoperative erythema, infection, scarring, and pigmentary alteration [6,7].

Tissue augmentation with collagen, hyaluronic acid, and autologous fat can be used to replace lost volume in large focal rolling [4,5] and distensible scars [2]. They offer transient improvement [4]. Among dermal fillers, it has been shown that hyaluronic acid-based products can stimulate endogenous collagen formation over time leading to sustained volumetric correction of scars. The administration of these fillers are not without risk [2].

For deep boxcar or large icepick scars, surgical approaches such as punch excision are the best treatments[1].

Subcision is an effective treatment for rolling scars. In this technique, the bleeding and subsequent clot formation result in elevation of the skin from the underlying scar tissue which induce neocollagenesis [5], and disrupt collagen fibers which anchor the superficial dermis to dermal and subdermal layers [2]. Hypertrophic scarring has been reported as its side effect. In comparison with dermal fillers, subcision is statistically more effective. Its combination with other modalities is more effective than subcision alone. It appears that subcision by loosening deep fibrous attachments can make acne scars more amenable to other treatment modalities [5].

Skin needling, as a collagen induction therapy, is effective in treating acne scaring by promoting neocollagenesis in a manner analogous to subcision and fractional ablative lasers [5].

We suggest saline therapy as effective and safe technique for treating atrophic acne scar. As a hypothesis, we suggest that the action mechanisms of our method include:

1. stimulating fibroblasts, and subsequently inducing collagen production and remodeling extracelluar matrix.

2. disrupting collagen fibers anchoring the superficial dermis to dermal and subdermal layers.

3. releasing fibrotic lesions which have appeared in response to inflammation secondary to acnein a manner analogous to subcision.

4. clot formation secondary to bleeding in the process of this technique results in elevation of the skin from the underlying scar tissue. It can work as a network for collagen and extracellular matrix neoformation.

5. release of the growth factors from white blood cells and platelets during this therapeutic method is effective in achieving positive response.

\section{Conclusion}

Our study revealed that the response to saline therapy was significant 
in the large pores and all types of the atrophic acne scars. In comparison with the severe acne scar, the patients with mild and moderate scars showed better responses. This study showed that the saline injection therapy can be an effective therapeutic option foratrophic acne scars, with no significant side effects. In addition, our study revealed that this procedure can successfully be used in thetreatment of patients suffering from the large skin pores.

\section{References}

1. O’Daniel TG (2011) Multimodal management of atrophic acne scarring in the aging face. Aesthetic Plast Surg 35: 1143-1150. [Crossref]

2. Munavalli GS, Smith S, Maslowski JM, Weiss RA (2013) Successful treatment of depressed, distensible acne scars using autologous fibroblasts: a multi-site, prospective, double blind, placebo-controlled clinical trial. Dermatol Surg 39: 1226-1236. [Crossref]
3. Kwok T, Rao J (2012) Laser management of acne scarring. Skin Therapy Lett 17: 4-6. [Crossref]

4. Taub AF, Garretson CB (2011) Treatment of Acne Scars of Skin Types II to V by Sublative Fractional Bipolar Radiofrequency and Bipolar Radiofrequency Combined with Diode Laser. J Clin Aesthet Dermatol 4: 18-27. [Crossref]

5. Hession MT, Graber EM (2015) Atrophic acne scarring: a review of treatment options. J Clin Aesthet Dermatol 8: 50-58. [Crossref]

6. Asilian A, Salimi E, Faghihi G, Dehghani F, Tajmirriahi N, et al. (2011) Comparison of Q-Switched 1064-nm Nd: YAG laser and fractional CO2 laser efficacies on improvement of atrophic facial acne scar. J Res Med Sci 16: 1189-1195. [Crossref]

7. Oh BH, Hwang YJ, Lee YW, Choe YB, Ahn KJ (2011) Skin characteristics after fractional photothermolysis. Ann Dermatol 23: 448-454. [Crossref]

8. Nirmal B, Pai SB, Sripathi H, Rao R, Prabhu S, et al. (2013) Efficacy and safety of erbium-doped yttrium aluminium garnet fractional resurfacing laser for treatment of facial acne scars. Indian J Dermatol Venereol Leprol 79: 193-198. [Crossref]

Copyright: $@ 2015$ Bagherani N. This is an open-access article distributed under the terms of the Creative Commons Attribution License, which permits unrestricted use, distribution, and reproduction in any medium, provided the original author and source are credited. 